\title{
Thalidomide-Then and Now: Case Report of a Woman With Thalidomide Embryopathy and Review of Current Thalidomide Uses
}

\author{
Sara Malik $^{1}$, Philip R. Cohen ${ }^{2}$ \\ 1. Feinberg School of Medicine, Northwestern University, Chicago, USA 2. Dermatology, University of California, Davis \\ Medical Center, Sacramento, USA
}

Corresponding author: Sara Malik, sara.malik@northwestern.edu

\begin{abstract}
Thalidomide was initially developed as a sedative; subsequently, its use was expanded to treat morning sickness in pregnant women. However, it was later discovered to be a teratogenic drug that was associated with embryopathy in women. A woman is described who was exposed to thalidomide in utero. She had several stigmata of thalidomide embryopathy. Although treatment of nausea and anxiety in pregnant women with thalidomide was discontinued in 1961, the drug has been found to be a useful agent for the management of several systemic conditions and dermatological disorders. Whether the treatment with thalidomide shall be incorporated in the therapeutic regime for patients with severe coronavirus disease 2019 (COVID-19) infection remains to be determined.
\end{abstract}

Review began 08/04/2021 Review ended 08/06/2021 Published 08/10/2021

๑) Copyright 2021 Malik et al. This is an open access article distributed under the terms of the Creative Commons Attribution License CC-BY 4.0., which permits unrestricted use, distribution, and reproduction in any medium, provided the original author and source are credited.
Categories: Dermatology

Keywords: contraception, covid-19, embryopathy, gestation, man, pregnancy, sars-cov-2, teratogen, thalidomide, woman

\section{Introduction}

Thalidomide is an anti-tumor necrosis factor-alpha drug. It was discovered in 1956 by researchers at the Chemie Grünenthal pharmaceutical company. It was released as a nonaddictive, nonbarbiturate sedative [1].

Initially, thalidomide was used by pregnant women with either nausea or anxiety, or both. In the childbearing market, its use expanded to treat insomnia in pregnant women. However, shortly thereafter, its use was associated with severe birth defects. The drug was discontinued for the treatment of pregnant women in 1961 in the United States medical market [2].

A 63-year-old woman with thalidomide embryopathy is described; her mother had been pregnant in 1957 and received the drug during her gestation. The features of thalidomide embryopathy are summarized. In addition, the current potential uses of thalidomide in medical therapy are reviewed.

\section{Case Presentation}

A 63-year-old woman presented for a total body skin examination. She had no prior history of skin cancer. However, her past medical history was significant for her mother receiving thalidomide during her pregnancy.

She had several stigmata of thalidomide embryopathy. These included strabismus, high-arched palate, and a right external ear that was smaller than the left external ear (Figures 1,2). She also had absent bones in her wrist, absent digits in her hands and no toes on her feet, shortened tibia and fibula, and hypermobility of her hip (Figures 3, 4). 


\section{Cureus}

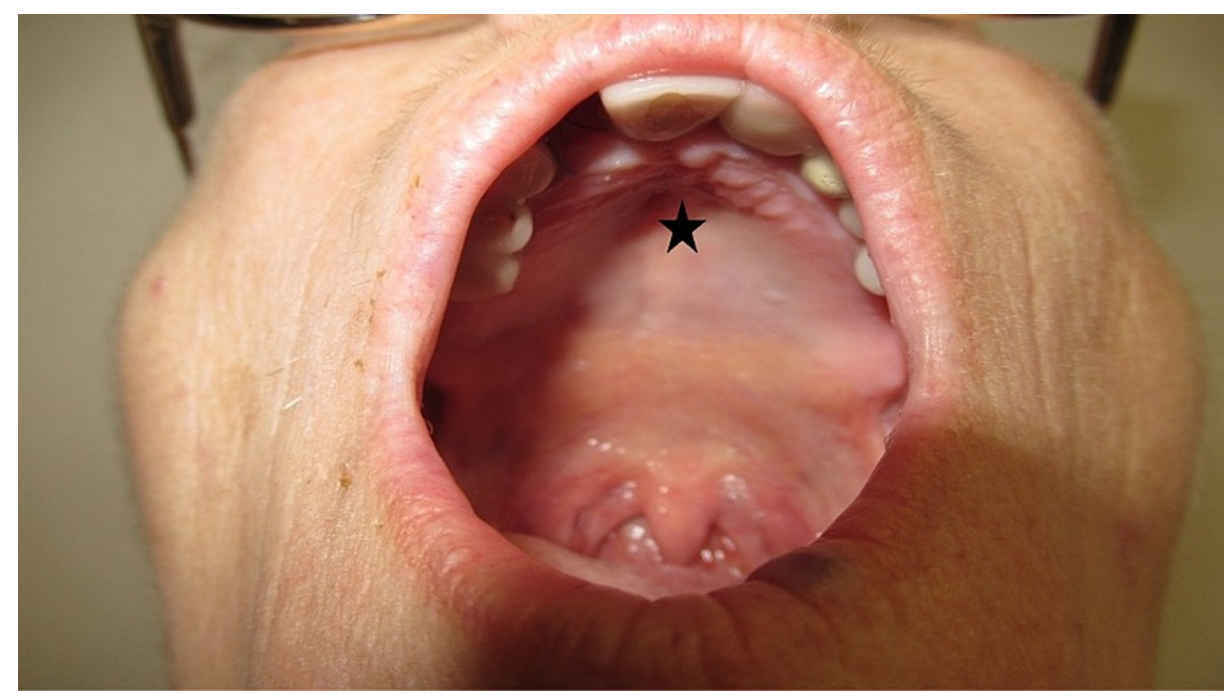

FIGURE 1: High-arched palate associated with exposure to thalidomide during gestation.

Oral examination of a 63-year-old woman demonstrates thalidomide exposure-associated high-arched palate (black star).
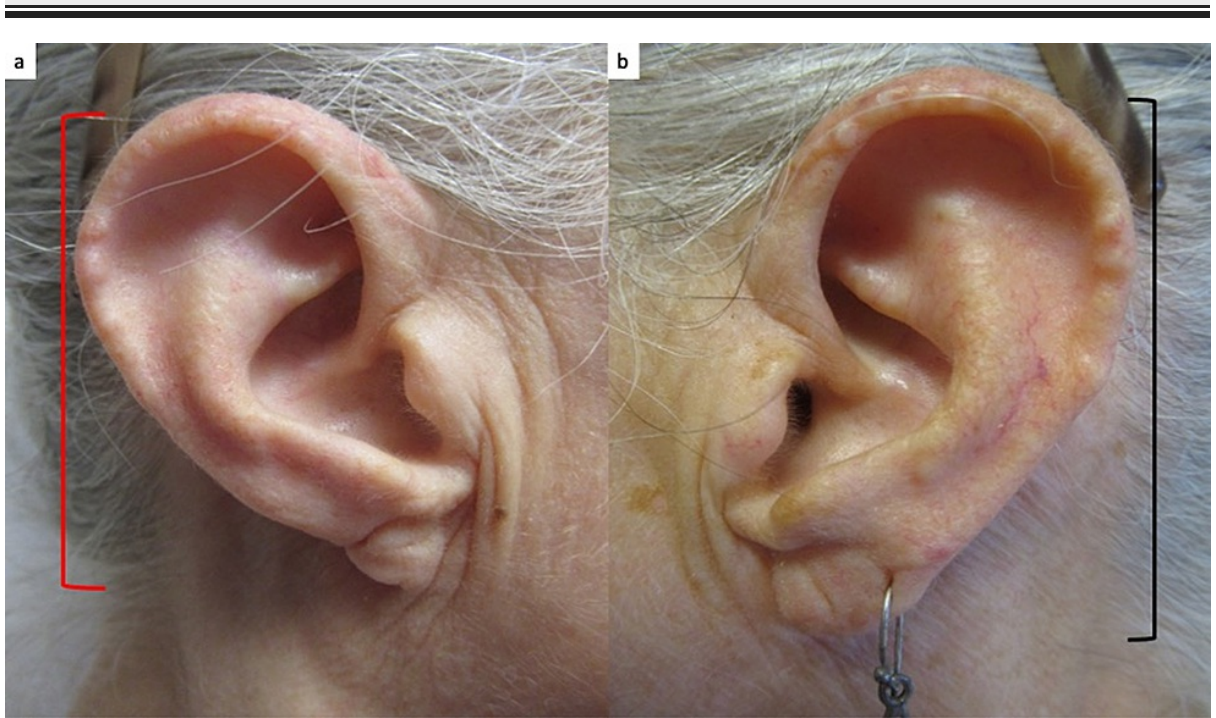

FIGURE 2: Ear abnormality in a woman with thalidomide embryopathy.

The length of the external ears is different. The length of the right external ear (red bracket) measures 2.5 centimeters (a) and the length of the left ear (black bracket) measures 3 centimeters (b). 


\section{Cureus}

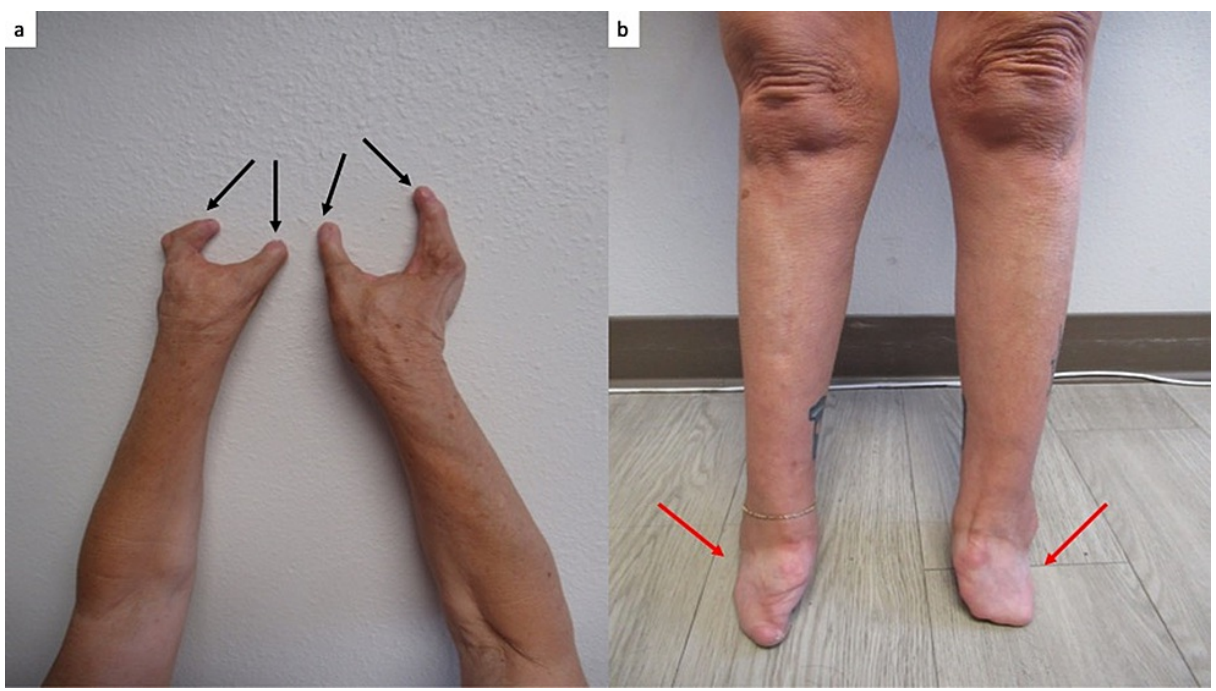

\section{FIGURE 3: Limb deformities of the upper and lower extremities associated with thalidomide exposure during gestation.}

A view of the extensor surface of the arms, from the elbows to the fingers, shows that each hand only has two digits (a); the black arrows point to each digit. The anterior view of the lower extremities of the legs, from the knees to the end of the feet (red arrows); there are no toes (b). The distance from the knees to ankles is shorter than normal.

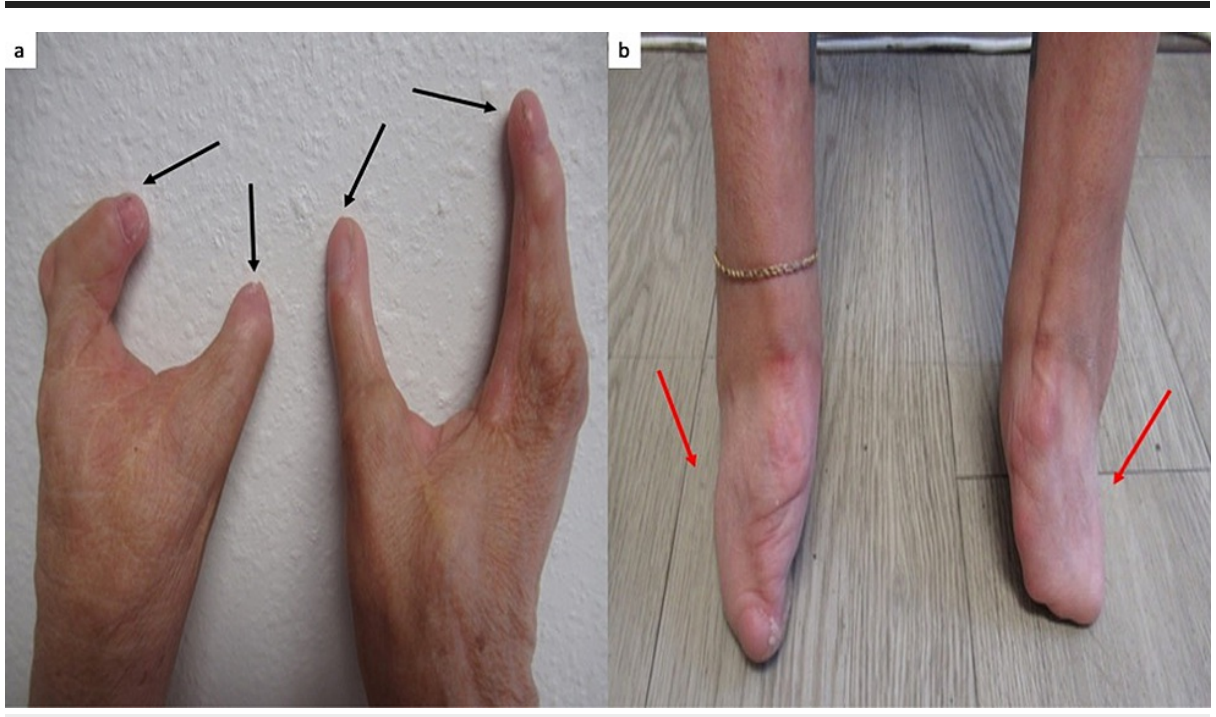

FIGURE 4: Embryopathy caused by thalidomide affecting the hands and feet.

Closer views of the hands (a) and feet (b) show two digits on each hand (black arrows) and feet without toes (red arrows); she has had multiple surgeries.

Cutaneous examination revealed multiple black and brown plaques on her back (Figure 5). Two of the black plaques on her back had changed in size and color; these were biopsied. Microscopic examination showed thickening of the epidermis (acanthosis) with horn cyst and heavy deposits of melanin in the basal layer of the epithelium; there was lymphocytic inflammation in the papillary dermis. Correlation of the clinical morphology and pathology findings established the diagnosis of benign irritated seborrheic keratosis. 


\section{Cureus}
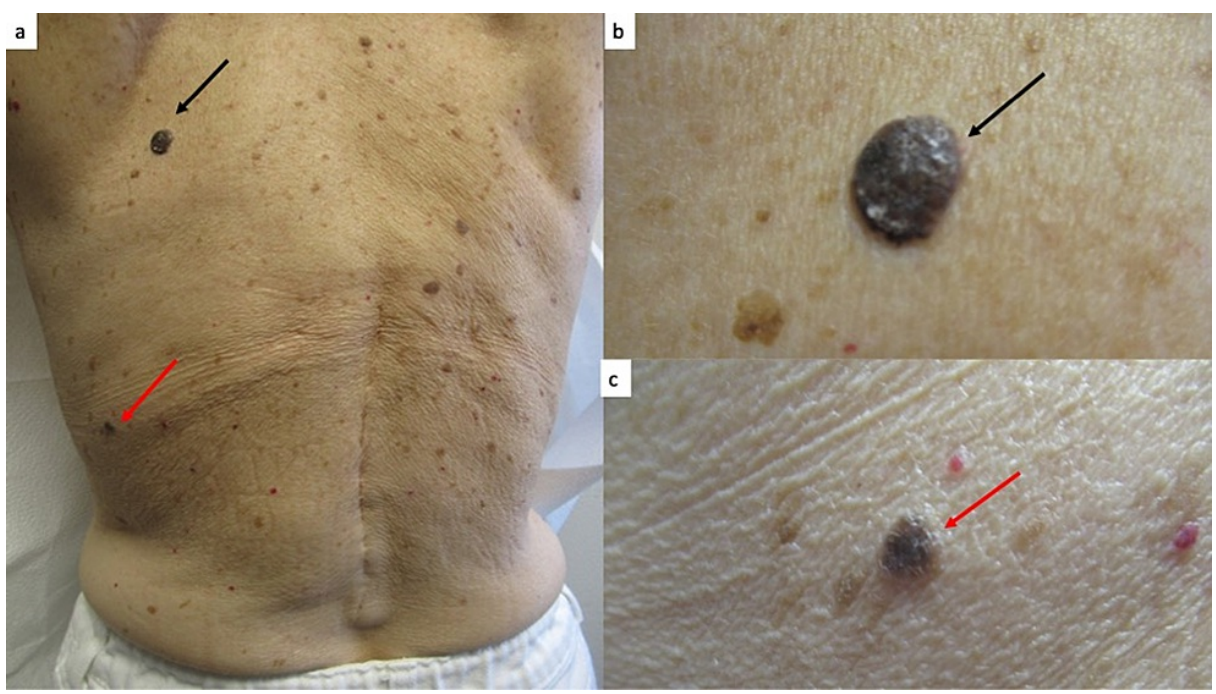

FIGURE 5: Seborrheic keratoses on the back.

Distant (a) and closer (b and c) views show several black plaques on the back of a 63-year-old woman. She had noticed changes in size and color of the lesions on the upper left back (black arrow, a and b) and midlateral left back (red arrow, a and c). The lesions were biopsied; microscopic examination established the diagnosis of a benign irritated seborrheic keratosis for both plaques.

\section{Discussion}

Thalidomide can cause thalidomide embryopathy; this occurs in pregnant women who receive the drug as early as 20 days after fertilization. The observed adverse sequelae depend on the gestational age at the time of exposure to the drug. For example, external ear defects were noted upon thalidomide intake between day 20 and day 24 after fertilization, limb defects between 24 and 30 days after conception, hip dislocation from 24 to 34 days, and lower limb defects were noted from 27 to 34 days after fertilization [1,2].

Thalidomide exposure can cause a wide range of defects. Some of the defects that are associated with thalidomide embryopathy include phocomelia in the upper and lower limbs, reduction in the length of the lower limbs, a more prominent shoulder joint, a hypoplastic or absent hip joint, defects in the eyes, ears, and face, vertebral column defects, and damage to the internal organs [2]. Our patient had multiple hallmarks of thalidomide embryopathy affecting the limbs, hip, mouth, eye, and ear.

Thalidomide is contraindicated in women who may become pregnant; two forms of contraception must be utilized in female patients one month prior, during, and for at least one month after discontinuing the drug. Currently, women of reproductive potential who are using the drug are registered in the THALIDOMID REMS ${ }^{\circledR}$ program. Women are required to complete a confidential survey monthly before a prescription can be written, and they must test negative in their pregnancy tests 24 hours before receiving a prescription. Furthermore, during the first four weeks, a pregnancy test needs to be performed weekly and then must be repeated every four weeks $[1,2]$.

Contraceptive use in men who plan to be sexually active while on thalidomide is also recommended. Although sperm-associated teratogenicity has not been definitively established, male patients should use contraception not only while taking the medication but also for at least one week after stopping the drug. Indeed, despite lacking evidence on the thalidomide's effects on male fertility, the System for Thalidomide Education and Prescribing Safety program has restricted the drug's marketing and use in men [3].

Several systemic and dermatologic conditions may potentially be treated with thalidomide (Table 1) [4-16]. The United States Food and Drug Administration has only approved thalidomide for the treatment of erythema nodosum leprosum and multiple myeloma. In addition to thalidomide being efficacious as an antineoplastic agent in patients with myeloma and lymphoma, thalidomide may be an efficacious treatment for inflammatory bowel disease in patients with Crohn's disease and possible therapeutic intervention for patients with fibrosing diseases such as pulmonary fibrosis and graft-versus-host disease. Similar to thalidomide, related analogs such as lenalidomide and pomalidomide have both been used in the treatment of cancer [4]. 


\section{Cureus}

\section{Condition $^{\mathrm{a}}$}

References

Actinic prurigo

Aphthous lesions (stomatitis and ulcers)

Behcet disease

Chronic graft-versus-host disease

COVID-19 infection ${ }^{b}$

Crohn's disease

Erythema multiforme

Erythema nodosum leprosum ${ }^{\mathrm{c}}$

Idiopathic pulmonary fibrosis

Immune complex vasculitis

Large B-cell lymphoma

Lichen planus

Lupus erythematosus (chronic discoid and systemic)

Multiple myeloma ${ }^{\mathrm{c}}$

[8]

Ophthalmopathy

Paraneoplastic pemphigus

Photodermatosis

Prurigo nodularis

Pyoderma gangrenosum

Rheumatoid arthritis

Sarcoidosis (prurigo-like lesions)

Scleroderma

[6]

Skin fibrosis

Other conditions ${ }^{\mathrm{d}, \mathrm{e}}$

\section{TABLE 1: Conditions potentially treated with thalidomide.}

Abbreviation: COVID-19, coronavirus disease 2019.

a Many of the conditions treated using thalidomide have been included; however, the table is not all-inclusive.

b The role of thalidomide in the treatment of COVID-19 has been postulated but not established.

${ }^{c}$ United Stated Food and Drug Administration approved condition for the treatment with thalidomide.

d Other conditions for which thalidomide has been used for treatment include bullous pemphigoid, histiocytosis, Jessner-Kanof disease, Kaposi sarcoma, leishmaniasis cutanea recidivans, necrobiosis lipoidica, scleromyxedema, and uremic pruritus.

${ }^{e}$ A possible use of thalidomide for managing cancer cachexia has been postulated. However, the investigators of a comprehensive systematic review concluded that there was inadequate evidence to recommend thalidomide for clinical practice for patients with advanced cancer who have cachexia.

Several mechanisms of action for thalidomide have been discovered, which include cytokine elaboration and an anti-inflammatory effect, T-cell and natural killer cell function and regulation, and anti-angiogenic properties [7,11]. Thalidomide can decrease the helper T-cell to suppressor T-cell ratio, which plays a role in 
the treatment of erythema nodosum leprosum. Furthermore, thalidomide's anti-angiogenic properties are associated with its ability to treat cancer [7]. Although the exact mechanism of thalidomide is unknown, thalidomide's different properties have allowed for its various potential uses in medical therapy.

The role of thalidomide in coronavirus disease 2019 (COVID-19) patients has not yet been established. Some investigators have proposed that thalidomide therapy may be efficacious in patients with a severe reaction to COVID-19 infection [10,17]. A study was performed in which patients with critical COVID-19 symptoms were enrolled in the thalidomide group and control group. Analysis was performed on six patients in each group. Patients in the thalidomide group were given thalidomide (100 milligrams daily for greater than or equal to seven days) in addition to the short-term administration of low-dose dexamethasone (40 milligrams intravenously at 12-hour intervals for three days; the dose was reduced and administered at 24-hour intervals for an additional five days) while patients in the control group were given short-term low-dose dexamethasone therapy. The study showed that thalidomide accelerated the negative conversion of severe acute respiratory syndrome coronavirus 2 (SARS-CoV-2), shortened the hospital stay, and decreased the requirement for mechanical ventilation in patients with severe COVID-19. The study revealed that in COVID-19-infected patients, thalidomide with low-dose glucocorticoid therapy was efficacious in reducing inflammation, inhibiting the production of inflammatory cytokines, and enhancing modulation of the immune system [17].

\section{Conclusions}

Thalidomide is a teratogenic drug that is associated with severe embryopathy when taken by pregnant women. The adverse events associated with its use during gestation are widespread. Although it was removed from the market in 1961, individuals who were exposed in utero can still be observed. Thalidomide is approved for use in leprosy patients who experience a post-treatment reaction of erythema nodosum leprosum. In addition, the drug is approved for the treatment of multiple myeloma. Moreover, there are several systemic and dermatologic conditions in which thalidomide has been demonstrated to be efficacious. Whether thalidomide has a role in the management of patients with COVID-19 remains to be established.

\section{Additional Information \\ Disclosures}

Human subjects: Consent was obtained or waived by all participants in this study. Conflicts of interest: In compliance with the ICMJE uniform disclosure form, all authors declare the following: Payment/services info: All authors have declared that no financial support was received from any organization for the submitted work. Financial relationships: Philip R. Cohen declare(s) Consultant from ParaPRO. Other relationships: All authors have declared that there are no other relationships or activities that could appear to have influenced the submitted work.

\section{References}

1. Asatsuma-Okumura T, Ito T, Handa H: Molecular mechanisms of the teratogenic effects of thalidomide . Pharmaceuticals (Basel). 2020, 13:95. 10.3390/ph13050095

2. Vargesson N: Thalidomide-induced teratogenesis: history and mechanisms. Birth Defects Res C Embryo Today. 2015, 105:140-56. 10.1002/bdrc.21096

3. Kumar P, Das A, Lal NR, Jain S, Ghosh A: Safety of important dermatological drugs (retinoids, immune suppressants, anti androgens and thalidomide) in reproductively active males with respect to pregnancy outcome: a brief review of literature. Indian J Dermatol Venereol Leprol. 2018, 84:539-46. 10.4103/ijdvl.IJDVL_77_17

4. Paghdal KV, Schwartz R: Thalidomide and its dermatologic uses. Acta Dermatovenerol Croat. 2007, 15:3944.

5. Faver IR, Guerra SG, Su WP, El-Azhary R: Thalidomide for dermatology: a review of clinical uses and adverse effects. Int J Dermatol. 2005, 44:61-7. 10.1111/j.1365-4632.2004.02445.x

6. Chen M, Doherty SD, Hsu S: Innovative uses of thalidomide. Dermatol Clin. 2010, 28:577-86. 10.1016/j.det.2010.03.003

7. Perri AJ 3rd, Hsu S: A review of thalidomide's history and current dermatological applications. Dermatol Online J. 2003, 9:5.

8. Ito T, Handa H: Molecular mechanisms of thalidomide and its derivatives. Proc Jpn Acad Ser B Phys Biol Sci. 2020, 96:189-203. 10.2183/pjab.96.016

9. Tiscornia JE, Poggio TV, Feinsilber DG, Coleman AE: Thalidomide should be tested as a therapeutic option in COVID-19 pneumonia. Skinmed. 2020, 18:254-5.

10. Goihman-Yahr M: Proposed use of thalidomide for the cytokine storm of COVID-19 . Clin Dermatol. 2020, 38:508. 10.1016/j.clindermatol.2020.04.015

11. Liu T, Guo F, Zhu X, He X, Xie L: Thalidomide and its analogues: a review of the potential for immunomodulation of fibrosis diseases and opthalmopathy. Exp Ther Med. 2017, 14:5251-7. 10.3892/etm.2017.5209

12. Upputuri B, Pallapati MS, Tarwater P, Srikantam A: Thalidomide in the treatment of erythema nodosum leprosum (ENL) in an outpatient setting: a five-year retrospective analysis from a leprosy referral centre in India. PLoS Negl Trop Dis. 2020, 14:e0008678. 10.1371/journal.pntd.0008678

13. Thangaraju P, Venkatesan S, Gurunthalingam M, Babu S, Tamilselvan T: Rationale use of thalidomide in erythema nodosum leprosum - a non-systematic critical analysis of published case reports. Rev Soc Bras 


\section{Cureus}

Med Trop. 2020, 53:e20190454. 10.1590/0037-8682-0454-2019

14. Ji D, Li Q, Cao J, et al.: Thalidomide enhanced the efficacy of CHOP chemotherapy in the treatment of diffuse large B cell lymphoma: a phase II study. Oncotarget. 2016, 7:33331-9. 10.18632/oncotarget.8973

15. Wang J, Zhang Y, Pan M: Thalidomide as a potential adjuvant treatment for paraneoplastic pemphigus: a single-center experience. Dermatol Ther. 2020, 33:e14353. 10.1111/dth.14353

16. Reid J, Mills M, Cantwell M, Cardwell CR, Murray LJ, Donnelly M: Thalidomide for managing cancer cachexia. Cochrane Database Syst Rev. 2012, 2012:CD008664. 10.1002/14651858.CD008664.pub2

17. Li Y, Shi K, Qi F, et al.: Thalidomide combined with short-term low-dose glucocorticoid therapy for the treatment of severe COVID-19: a case-series study. Int J Infect Dis. 2021, 103:507-13.

10.1016/j.ijid.2020.12.023 